\title{
Multipeaked polarons in soft potentials
}

\author{
M. A. Fuentes, ${ }^{1}$ P. Maniadis, ${ }^{2,3}$ G. Kalosakas, ${ }^{4}$ K. Ø. Rasmussen, ${ }^{4}$ A. R. Bishop,${ }^{4}$ V. M. Kenkre, ${ }^{1}$ and Yu. B. Gaididei ${ }^{5}$ \\ ${ }^{1}$ Consortium of the Americas for Interdisciplinary Science, Department of Physics and Astronomy, \\ University of New Mexico, Albuquerque, New Mexico 87131, USA \\ and CONICET, Argentina \\ ${ }^{2}$ LADIR-CNRS, UMR 7075 Université Pierre et Marie Curie, 2 rue Henry Dunant, 94320 Thiais, France \\ ${ }^{3}$ Laboratoire Léon Brillouin (CEA-CNRS), CEA Saclay 91191-Gif-sur-Yvette Cedex, France \\ ${ }^{4}$ Theoretical Division and Center for Nonlinear Studies, Los Alamos National Laboratory, Los Alamos, New Mexico 87545, USA \\ ${ }^{5}$ Bogolyubov Institute for Theoretical Physics, 14-b Metrologichna Strasse, 252143 Kiev, Ukraine \\ (Received 10 July 2003; revised manuscript received 2 February 2004; published 10 August 2004)
}

\begin{abstract}
We consider a minimal coupled charge/excitation-lattice model capturing a competition between linear polaronic self-trapping and the self-focusing effects of a soft nonlinear on-site potential. The standard singlehumped polaron ceases to exist above a critical value of the coupling strength, closely related to the inflection point in the nonlinear potential. For couplings beyond this critical value, we find that successive multihumped polaronic solutions correspond to the lowest-energy stationary states of the system, which may admit interesting quantum resonance behavior.
\end{abstract}

DOI: 10.1103/PhysRevE.70.025601

PACS number(s): 05.45.- a, 45.90.+t

\section{INTRODUCTION}

One of the most intriguing current problems in the physics of soft matter is the understanding of the mechanisms controlling charge and energy localization and transport in bio- and synthetic polymers. For example, it is generally thought that understanding these mechanisms will provide the key to better comprehend and technologically exploit the speed and efficiency of the important biological process of photosynthesis [1]. Similarly, charge localization and transport properties play a determining role in the more technologically mature field of photogeneration and transport in conjugated polymers [2].

The notion that effective nonlinearity due to the interaction between charge or excitation and vibrational degrees of freedom can result in structurally very stable excitations led Davydov several decades ago to propose that solitonlike excitations might be efficient agents of energy and charge transfer in biological molecules [3,4]. While such transfer mechanisms are conceptually appealing, subsequent work [5] has exposed several practical flaws. Nonetheless, intrinsic localized excitations due to interacting fields have been demonstrated in strongly nonlinear materials, especially in regimes where lattice discreteness and localization scales are comparable $[4,6-8]$.

Our aim here is to propose a more general concept, relevant to a wide range of soft matter applications, which is closely related to intrinsic localization. Intrinsic localization due to interacting degrees of freedom is well studied in the framework of linear trapping, as, for example, found in the Holstein model $[9,10]$. This may result in single- and also multiple-humped polarons and excitons [11]. However, in that case the single-humped polaron always exists as the ground state of the system. In this work, we introduce the idea of linear self-trapping with the additional effect of a "soft" nonlinear potential. The coexistence of these two effects can produce stable polaronic multihumped complexes as the lower-energy stationary states, which may facilitate novel mechanisms for transport assisted by resonant tunneling [12]. We will show that the presence of soft nonlinearity in the potential plays a determining role in the existence of the single and multihumped solutions. In particular, the inflection point of the nonlinear potential determines a threshold for multihumps polarons; for amplitudes below the inflection point, the nonlinearity is responsible for weaker anharmonic renormalization effects.

To most clearly expose the concepts, we study here a minimal nonlinear model to describe "softness" that admit the above novel polaroniclike behavior. In dimensionless units, the model can be written in terms of the coupled Hamiltonian

$$
\begin{aligned}
H= & \sum_{n}\left\{-J \varphi_{n}^{*}\left(\varphi_{n+1}+\varphi_{n-1}\right)-\chi y_{n}\left|\varphi_{n}\right|^{2}+V\left(y_{n}\right)\right. \\
& \left.+\frac{k}{2}\left(y_{n+1}-y_{n}\right)^{2}+\frac{1}{2}\left(\frac{d y_{n}}{d t}\right)^{2}\right\}
\end{aligned}
$$

where $V(y)$ represent an anharmonic on-site potential of the form

$$
V(y)=D[\exp (-\alpha y)-1]^{2} .
$$

In Eq. (1), the lattice site index $n$ runs from 1 to $N$, with $N$ the total number of sites, $\varphi_{n}$ is the probability amplitude for the charge/excitation located at the $n$th site, $y_{n}$ is the dimensionless lattice displacement at this site, $J$ is the dimensionless transfer integral, $k$ is the dimensionless lattice spring constant, and $\chi$ is the dimensionless coupling constant between the interacting fields. We consider the semiclassical equations of motion $[10,13]$ derived from the Hamiltonian (1); viz., we treat the charge quantum mechanically and the vibrational motion classically. The Schrödinger equation then becomes 


$$
i \frac{d \varphi_{n}}{d t}=-J\left(\varphi_{n+1}+\varphi_{n-1}\right)-\chi y_{n} \varphi_{n} .
$$

Newton's equations of motion for the displacements $y_{n}$ become

$$
\frac{d^{2} y_{n}}{d t^{2}}=-V^{\prime}\left(y_{n}\right)+\chi\left|\varphi_{n}\right|^{2}+k\left(y_{n+1}-2 y_{n}+y_{n-1}\right) .
$$

The prime denotes differentiation with respect to $y_{n}$, while $t$ represents dimensionless time.

We are interested here in stationary polarons where the wave vector $\varphi_{n}$ oscillates with a frequency $\omega_{0}$, while the lattice is at rest, i.e.,

$$
\varphi_{n}(t)=\Psi_{n} \exp \left(-i \omega_{0} t\right), \quad \frac{d^{2} y_{n}}{d t^{2}}=\frac{d y_{n}}{d t}=0 .
$$

Polarons, in this context, become more localized and accompanied by larger lattice displacement when the coupling $\chi$ becomes stronger. An intuitive understanding of the interesting properties of the system arises in the limit of uncoupled lattices sites, known as the "anticontinuous limit" $[11,14]$. This is realized with $J=0$ and $k=0$. In this limit, the singlehumped polaron is localized on a single lattice site, say $n_{0}$, i.e., $\Psi_{n}=\delta_{n, n_{0}}$. Solving the corresponding Eq. (4), under the condition (5), we find the lattice displacements

$$
y_{n_{0}}^{ \pm}=y_{1}^{ \pm}=-\frac{1}{\alpha} \ln \left(\frac{1 \pm \sqrt{1-\frac{2 \chi}{D \alpha}}}{2}\right) \text {. }
$$

The stability of these two $( \pm)$ solutions is determined by the following set of equations:

$$
\begin{gathered}
\frac{d r_{n_{0}}}{d t}=0, \quad \frac{d \varpi_{n_{0}}}{d t}=\chi y_{n_{0}}\left(1+\varpi_{n_{0}}^{2}\right), \\
\frac{d y_{n_{0}}}{d t}=u_{n_{0}}, \quad \frac{d u_{n_{0}}}{d t}=-V^{\prime}\left(y_{n_{0}}\right)+\chi r_{n_{0}},
\end{gathered}
$$

which have been obtained by linearizing around the solutions (6). We have defined

$$
\varpi_{n_{0}}=\frac{\operatorname{Im}\left[\varphi_{n_{0}}\right]}{\operatorname{Re}\left[\varphi_{n_{0}}\right]}, \quad r_{n_{0}}=\left|\varphi_{n_{0}}\right|^{2} .
$$

In these variables, the stationary single-humped polaron of the anticontinuous limit is (we omit the notation $n_{0}$ from now on) $r_{s}=1, \dot{y}_{s}=u_{s}=0, y_{s}=y_{1}^{ \pm}$, Eq. (6), and the phase is arbitrary. The stability is determined by the eigenvalues of the following eigenvalue problem:

$$
\left(\begin{array}{ccc}
0 & 0 & 0 \\
0 & 0 & 1 \\
\chi & -V^{\prime \prime}\left(y_{s}\right) & 0
\end{array}\right)\left(\begin{array}{l}
\delta r \\
\delta y \\
\delta u
\end{array}\right)=\lambda\left(\begin{array}{c}
\delta r \\
\delta y \\
\delta u
\end{array}\right) .
$$

(The dynamics of $\varpi$ is irrelevant, and is ignored.) Here, $V^{\prime \prime}\left(y_{s}\right)$ is the second derivative of the on-site potential. Diagonalization of this matrix yields the eigenvalues
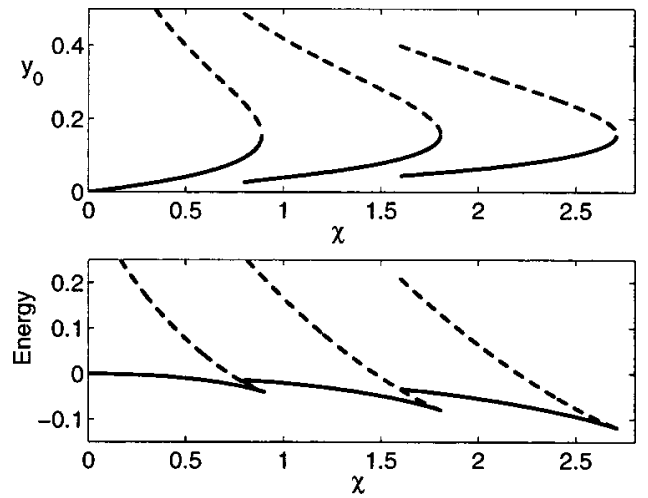

FIG. 1. Polaron solutions at the anticontinuous limit. Left curves represent single-humped stationary solutions while middle and right curves represent double- and tripled-humped solutions, respectively. Upper panel: Amplitude of stable (solid line) and unstable (dashed line) stationary states according to Eq. (13) [for $s=1$ (singlehumped), $s=1 / 2$ (double-humped), and $s=1 / 3$ (triple-humped)]. Lower panel: Energy of stable (solid line) and unstable (dashed line) stationary states. Parameters values are $D=0.4, \alpha=4.45, J=k$ $=0$.

$$
\lambda_{0}=0, \quad \lambda_{1,2}= \pm \sqrt{-V^{\prime \prime}\left(y_{s}\right)} .
$$

We see that $y_{1}^{+}$is unstable, as a result of the positive eigenvalue $\lambda_{1}\left[V^{\prime \prime}\left(y_{1}^{+}\right)<0\right]$. The stationary solution $y_{1}^{-}$is a center, having imaginary eigenvalues $\left[V^{\prime \prime}\left(y_{1}^{-}\right)>0\right]$.

From Eq. (6), the maximum amplitude for the stable $y_{1}^{-}$ occurs when the two solutions become equal,

$$
y^{+}=y^{-}=\frac{\ln 2}{\alpha}=y_{\text {inf }},
$$

corresponding to the inflection point of the on-site potential, $V(y)$. In this sense, the inflection point plays a determining role for the existence of the stable single-humped stationary solution, which, as long as it exists, corresponds to the lowest-energy stationary state of the system. The stable and unstable solutions are situated symmetrically with respect to this point and the maximum amplitude of the stable stationary lattice displacement $y_{n}$ corresponds precisely to its value (see Fig. 1). Qualitatively similar behavior is expected for the stationary, more spatially extended solution of Eqs. (3) and (4) (see below). Another important result of this analysis is that these solutions only exist for $\chi$ below a critical value [see Eq. (6)]

$$
\chi \leqslant \chi_{c} \equiv \frac{D \alpha}{2} .
$$

Relaxing the condition $\Psi_{n}=\delta_{n, n_{0}}$, and setting $\Psi_{n}$ $=\sqrt{\varsigma} \delta_{|n|, n_{0}}$ with $\varsigma=0.5$ (representing a situation with a doublepeaked polaron at the positions $n= \pm n_{0}$, respectively), the double-humped stationary solution for the amplitude in the lattice becomes 


$$
y_{2}^{ \pm}=-\frac{1}{\alpha} \ln \left(\frac{1 \pm \sqrt{1-\frac{2 \chi \varsigma}{D \alpha}}}{2}\right) \text {, }
$$

where the subscript 2 denotes the double-humped polaron character of this solution. The critical value, $\chi_{c}(2)$, of $\chi$ in this case is

$$
\chi_{c}(2) \equiv \frac{D \alpha}{2 \varsigma}=2 \chi_{c},
$$

with $\chi_{c}$ given by Eq. (12). This procedure can be extended to multihumped localized solutions. The critical coupling parameter $\chi_{c}(m)$ for $m$-humped stationary solutions will follow the relation

$$
\chi_{c}(m)=m \chi_{c} .
$$

The results derived in the anticontinuous limit are illustrated in Fig. 1, which shows the amplitude and energy of the described stationary polaronic solutions as a function of $\chi$. It is interesting to note from this figure that the topology of the lowest-energy stationary state depends on $\chi$, in the sense that once the single-humped polaron disappears, the doublehumped polaron becomes the lowest-energy stationary state, and so on [15]. From this analysis, we expect that in the extended model, Eqs. (3) and (4), the solution for a single polaron will disappear above a given $\chi$ and then only stationary states with a larger number of humps will exist.

For departures from the anticontinuous limit, it is easy to see that the coupling to the nearest neighbors will cause an effective decrease in the on-site potential. This change will move the maximum amplitude for the deformation of the lattice such that the maximum amplitude $y_{0}$ of the singlehumped stationary solution will be $y_{0}>\ln 2 / \alpha$, i.e., greater than $y_{\text {inf }}$, but the basic scenario will be as described in the anti-continuous limit.

We now present numerical results for the complete set of Eqs. (3) and (4) in a case away from the anticontinuous limit. To obtain the stationary polaron solutions, we apply the method detailed in Ref. [16].

In Fig. 2, we see the typical shape of a single-humped polaron solution (upper two panels) for a given $\chi$. As predicted, we find that this stationary solution exhibits a bifurcation behavior depending on the coupling constant $\chi$. The single-humped stationary solution disappears above a critical value of the coupling parameter, and then only polarons with two or more humps can exist. This is in agreement with the analytical results derived at the anticontinuous limit. In Fig. 2, double- (middle two panels) and triple- (bottom panels) humped stationary solutions are also shown.

Figure 3 shows the polaron energies as a function of coupling constant $\chi$. Again we see that the topology of the lowest-energy stationary state depends on the value of $\chi$, for single-, double-, and triple-humped polarons. Figure 4 shows the maximum amplitude in the lattice as a function of coupling constant $\chi$. The maximum amplitude for these multihumped polaron solutions is larger than the inflection point for the on-site potential for the set of parameters used due to the nearest neighbor interactions on the lattice.

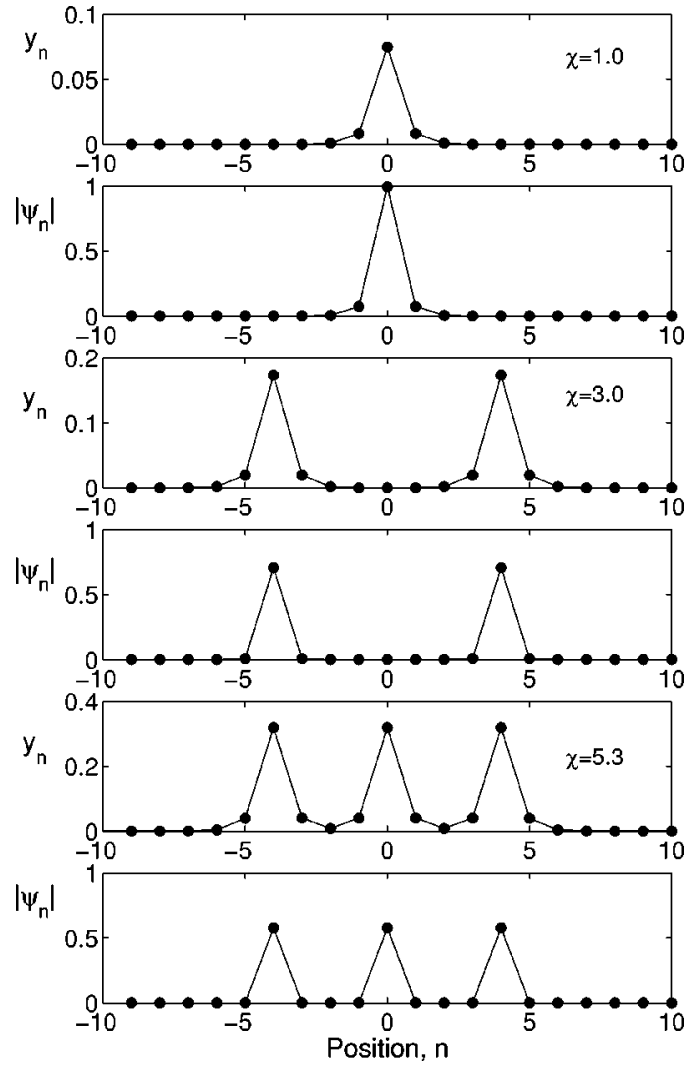

FIG. 2. Polaron solutions with one, two, and three humps. Both lattice, $y_{n}$, and charge/excitation, $\left|\Psi_{n}\right|$, are shown. The other parameters are $D=0.4, \alpha=4.45, J=0.005$, and $k=2.0$.

We emphasize that the observed behavior is due to the fact that the lattice displacements are on the soft part of the on-site potential. For the polaron case studied here, this requires a positive coupling constant $\chi$. For negative values of $\chi$, the system does not exhibit such a succession of multihumped polarons [16], since there is no softness (and, therefore, inflection point) in the other part of the on-site potential (for negative displacements). It is interesting that although the system is quite far from the anticontinuous limit, the various polarons cease to exist when distortion of the lattice

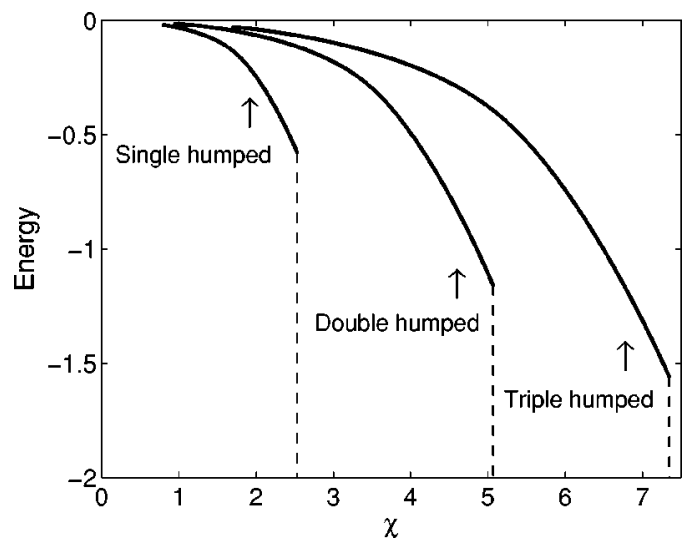

FIG. 3. Energy as a function of $\chi$ for single-, double-, and triplehumped stationary polaronic solutions, respectively. The parameters are as in Fig. 2. 


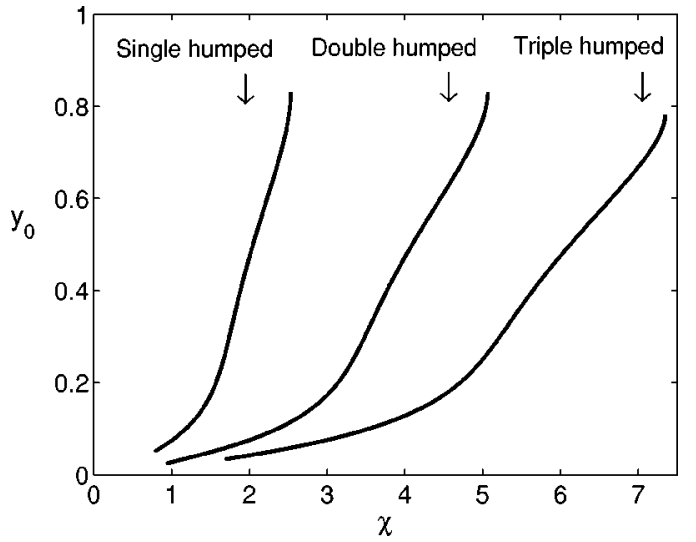

FIG. 4. Maximum amplitude in the lattice, $y_{0}$, as a function of $\chi$, for the single-, double-, and triple-humped polaron solutions, respectively. The parameters are as in Fig. 2.

of a certain amplitude is reached, regardless of the number of humps. Further, we see that relation (15) between the critical $\chi$ values still is approximately valid for these cases.

In summary, we have considered a minimal model to describe novel polaronic effects in soft matter. Due to the coexistence of the linear polaronic trapping and the softness of the on-site potential, we have found that the familiar singlehump polaron solution disappears above a critical value of the coupling constant $\chi$. The inflection point has been shown to play a determining role for the existence and stability of this polaronic state, thereby providing an operational definition of "softness." Also, we have found numerically that multihumped polaron states can coexist for a small range of the parameters. The structure of the lower-energy stationary states, therefore, depends on $\chi$ [15], and there are critical values of this parameter beyond which the lowest-energy stationary state becomes a polaron with additional humps, corresponding to self-focusing in the nonlinear lattice potential. We note that the standard polaronic localization allows exploration of the lattice nonlinearity (in the polaron vicinity) and formation of the multihumped excitations if the lattice is sufficiently soft. Even a hard nonlinear potential will admit such excitations, but only if sufficient energy is introduced locally [17]. Ongoing studies show a complex interaction between the humps in a multihumped polaron structure and the existence of charge/excitation exchange between humps in the form of "tunneling," similarly to Ref. [12]. These behaviors suggest that the introduction of an appropriate resonance field may enhance the transport properties of these novel complex solutions, providing efficient transport in soft materials [18].

Research at Los Alamos National Laboratory is supported by the U.S. DOE under Contract No. W-7405-ENG-36. V.M.K. acknowledges partial support of the NSF under Grant No. DMR-0097204. One of us (P.M.) was supported by the European TMP program LOCNET, Grant No. HPRNCT-1999-00163.
[1] X. Hu et al., Q. Rev. Biophys. 35, 12002.

[2] Primary Photoexcitations in Conjugated Polymers: Molecular Exciton Versus Semiconductor Band Model, edited by N. S. Sariciftci (World Scientific, Singapore, 1997); A. J. Heeger, MRS Bull. 26, 900 (2001)

[3] A. S. Davydov, J. Theor. Biol. 38, 559 (1973); 66, 379 (1977); Solitons in Molecular Systems (Reidel Publishing Company, Boston, 1985).

[4] A. C. Scott, Phys. Rep. 217, 1 (1992).

[5] P. S. Lomdahl and W. C. Kerr, Phys. Rev. Lett. 55, 1235 (1985); X. D. Wang, D. W. Brown, and K. Lindenberg, ibid. 62, 1796 (1989); D. Vitali, P. Allegrini, and P. Grigolini, Chem. Phys. 180, 297 (1994); M. I. Salkola et al., Phys. Rev. B 52, R3824 (1995).

[6] K. S. Song and R. T. Williams, Self-Trapped Exitons, 2nd ed., Springer Series in Solid State Sciences Vol. 105 (Springer, New York, 1996).

[7] D. M. Alexander and J. A. Krumhansl, Phys. Rev. B 33, 7172 (1986); M. Barthes, G. De Nunzio, and M. Ribet, Synth. Met. 76, 337 (1996); J. Edler, P. Hamm, and A. C. Scott, Phys. Rev. Lett. 88, 067403 (2002).

[8] B. I. Swanson et al., Phys. Rev. Lett. 82, 3288 (1999); N. K. Voulgarakis et al., Phys. Rev. B 64, 020301 (2001); G. Kalosakas, A. R. Bishop, and A. P. Shreve, Phys. Rev. B 66,
094303 (2002).

[9] T. Holstein, Ann. Phys. (N.Y.) 8, 325 (1959).

[10] G. Kalosakas, S. Aubry, and G. P. Tsironis, Phys. Rev. B 58, 3094 (1998).

[11] S. Aubry, G. Abramovici, and J.-L. Raimbault, J. Stat. Phys. 67, 675 (1992).

[12] Y. B. Gaididei, P. L. Christiansen, and S. M. Mingaleev, Phys. Scr. 51, 289 (1995).

[13] S. Komineas, G. Kalosakas, and A. R. Bishop, Phys. Rev. E 65, 061905 (2002); G. Kalosakas, K. Ø. Rasmussen, and A. R. Bishop, J. Chem. Phys. 118, 3731 (2003).

[14] S. Aubry, Physica D 71, 196 (1994).

[15] The discussed (multihumped) polarons represent the lowerenergy stationary states of the system, but large-amplitude unbound nonstationary solutions can have lower energies.

[16] P. Maniadis, G. Kalosakas, K. Ø. Rasmussen, and A. R. Bishop, Phys. Rev. B 68, 174304 (2003).

[17] W. Z. Wang et al., Phys. Rev. Lett. 80, 3284 (1998).

[18] This mechanism, similar to that of Ref. [12], is different from the softening of a pinning mode that is responsible for enhanced mobility in Holstein-Hubbard many-electron systems. See, for example, L. Proville and S. Aubry, Eur. Phys. J. B 11, 41 (1999). 